OPEN ACCESS

Edited by:

Diana Elizabeth Marco,

Consejo Nacional de Investigaciones

Cientificas y Técnicas (CONICET),

Argentina

Reviewed by:

Alfonso Benítez-Páez,

Instituto de Agroquímica y Tecnología

de Alimentos (IATA), Spain

David Correa Galeote,

Universidad Nacional Autónoma

de México, Mexico

*Correspondence:

Jun Xu

xujunn@sjtu.edu.cn

tThese authors have contributed equally to this work

Specialty section:

This article was submitted to

Aquatic Microbiology,

a section of the journal

Frontiers in Microbiology

Received: 21 September 2017

Accepted: 22 May 2018

Published: 07 June 2018

Citation:

Wang J, Lu Y, Nawaz MZ and Xu J (2018) Comparative Genomics

Reveals Evidence of Genome Reduction and High Extracellular

Protein Degradation Potential in Kangiella. Front. Microbiol. 9:1224. doi: 10.3389/fmicb.2018.01224

\section{Comparative Genomics Reveals Evidence of Genome Reduction and High Extracellular Protein Degradation Potential in Kangiella}

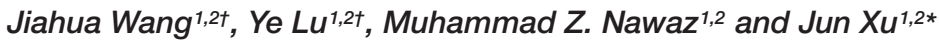 \\ 1 Institute of Oceanography, Shanghai Jiao Tong University, Shanghai, China, ${ }^{2}$ State Key Laboratory of Microbial \\ Metabolism, School of Life Sciences and Biotechnology, Shanghai Jiao Tong University, Shanghai, China
}

The genus Kangiella has recently been proposed within the family Kangiellaceae, belonging to order Oceanospirillales. Here, we report the complete genome sequence of a novel strain, Kangiella profundi FT102, which is the only Kangiella species isolated from a deep sea sediment sample. Furthermore, gaps in the publicly available genome scaffold of $K$. aquimarina DSM 16071 (NCBI Reference Sequence: NZ_ARFE00000000.1) were also filled using polymerase chain reaction (PCR) and Sanger sequencing. A comparative genomic analysis of five Kangiella and 18 nonKangiella strains revealed insights into their metabolic potential. It was shown that low genomic redundancy and Kangiella-lineage-specific gene loss are the key reasons behind the genome reduction in Kangiella compared to that in any other freeliving Oceanospirillales strain. The occurrence of relatively diverse and more frequent extracellular protease-coding genes along with the incomplete carbohydrate metabolic pathways in the genome suggests that Kangiella has high extracellular protein degradation potential. Growth of Kangiella strains has been observed using amino acids as the only carbon and nitrogen source and tends to increase with additional tryptone. Here, we propose that extracellular protein degradation and amino acid utilization are significant and prominent features of Kangiella. Our study provides more insight into the genomic traits and proteolytic metabolic capabilities of Kangiella.

Keywords: marine bacteria, Oceanospirillales, Kangiella, genome reduction, protein degradation

\section{INTRODUCTION}

The ocean covers $71 \%$ of the earth's surface and is regarded as the largest habitat for life on the planet Earth. Marine microorganisms are known to play an essential role in energy conservation and biogeochemical cycling in the oceans. Heterotrophic prokaryotes are considered key players in the decomposition of the dissolved organic matter (DOM) and particulate organic matter (POM) present therein (DeLong and Karl, 2005; Azam and Malfatti, 2007).

Oceanospirillales is an order of proteobacteria with seven families of heterotrophic marine bacteria that are usually associated with oil spills and are known to be involved in xylan and hydrocarbon utilization (Choi et al., 2012; Cao et al., 2014). Recently, a new family named Kangiellaceae has been proposed within the order Oceanospirillales based on phylogenetic, 
chemotaxonomic and physiological characteristics; this family comprises three genera: Kangiella, Aliikangiella, and Pleionea (Wang et al., 2015). Although the Kangiella genus was reclassified within Kangiellaceae instead of Alcanivoracaceae based on $16 \mathrm{~S}$ rRNA gene phylogeny (Wang et al., 2015), taxonomic signatures at the genomic level are still needed.

Kangiella are gram-negative long rods that are non-motile and non-spore-forming bacteria. Nine strains belonging to the genus Kangiella have been isolated from various marine environments, including marine sand (Kim et al., 2015), tidal flat sediments and coastal regions (Yoon et al., 2004, 2012; Romanenko et al., 2010; Ahn et al., 2011; Jean et al., 2012; Lee et al., 2013). For example, both K. koreensis DSM 16069 and Kangiella aquimarina DSM 16071 were isolated from tidal flat sediment at Daepo Beach, Yellow Sea, Korea, and were shown to grow optimally at 30$37^{\circ} \mathrm{C}$ and $\mathrm{pH} 7.0-8.0$ (Yoon et al., 2004). K. sediminilitoris KCTC 23892 was isolated from tidal flat sediment from the South Sea in South Korea, which was shown to grow optimally at $30-37^{\circ} \mathrm{C}$ and pH 7.0-7.5 (Lee et al., 2013). Kangiella geojedonensis KCTC 23420 was isolated from seawater off the southern coast of Korea, and was shown to grow optimally at $10-40^{\circ} \mathrm{C}$ and $\mathrm{pH} 7.0-7.5$ (Yoon et al., 2004). Recently, we isolated a novel strain named Kangiella profundi from a deep sea sediment sample that was collected from the southwest Indian Ocean at a depth of $2784 \mathrm{~m}$ (Xu et al., 2015). Due to limited research, our understanding about the metabolic potential and ecological functions of bacteria belonging to the family Kangiellaceae is still obscure.

To date, no genome has been completely sequenced from either of the two genera Aliikangiella and Pleionea. Moreover, only three completely sequenced genomes of the genus Kangiella, i.e., K. koreensis DSM 16069 (GenBank accession: GCA_000024085.1), K. sediminilitoris KCTC 23892 (GenBank accession: GCA_001708405.1), and K. geojedonensis KCTC 23420 (GenBank accession: GCA_000981765.1), are available. In this study, we report the complete genome sequence of $K$. profundi FT102 (GenBank accession: CP025120). Comparative genomicsbased approaches were utilized to explore the metabolic potential of Kangiella. We show that featured genomic reduction in Kangiella is due to low genomic redundancy as well as Kangiella lineage-specific gene loss. The metabolic base of Kangiella as a powerful extracellular protein degrader was investigated. Our study provides the first insights into the genomic and metabolic capabilities of Kangiella.

\section{MATERIALS AND METHODS}

\section{Bacterial Strains and Growth Conditions}

Kangiella profundi FT102 ${ }^{T}$ ( = CGMCC $1.12959^{T}=$ KCTC $42297^{T}=\mathrm{JCM} 30232^{T}$ ) is from our laboratory stock. The strains K. koreensis DSM 16069 (JCM 12317), K. aquimarina DSM 16071 (JCM 12318) and K. geojedonensis KCTC 23420 were obtained from Japan Collection of Microorganisms (JCM) and Korean Collection for Type Cultures (KCTC). These Kangiella strains were grown in marine broth 2216 (Xu et al., 2015) and marine peptone medium ( $1 \mathrm{~L}$ of broth containing $1 \mathrm{~g}$ of yeast extract, $10 \mathrm{~g}$ of tryptone, and $1000 \mathrm{~mL}$ of artificial sea water; $\mathrm{pH}$ adjusted to 7.3 ) at $37^{\circ} \mathrm{C}$ and $200 \mathrm{rpm}$ under aerobic conditions. Bacterial growth was measured by an automatic turbiditimetry using a Bioscreen C analyzing system (Labsystems).

\section{Cultivation in Defined Medium}

The defined cultivation method for Kangiella has been described elsewhere (Widdel et al., 2006). All 20 common amino acids, as a sole source of carbon, nitrogen and energy, were filter-sterilized and added to the media at a final concentration of $0.2 \mathrm{~g} / \mathrm{L}$ of each amino acid. The medium contained (g/L): $\mathrm{NaCl}(26.0)$, $\mathrm{MgCl}_{2} \cdot 6 \mathrm{H}_{2} \mathrm{O}$ (5.0), $\mathrm{CaCl}_{2} \cdot 2 \mathrm{H}_{2} \mathrm{O}$ (1.4), $\mathrm{NH}_{4} \mathrm{Cl}(0.3), \mathrm{KH}_{2} \mathrm{PO}_{4}$ (0.1), $\mathrm{KCl}(0.50), \mathrm{NaNO}_{3}(1.0)$, with (mg/L), EDTA, disodium salt (5.20), $\mathrm{FeSO}_{4} \cdot 7 \mathrm{H}_{2} \mathrm{O}(2.10)$, and $(\mu \mathrm{g} / \mathrm{L}): \mathrm{H}_{3} \mathrm{BO}_{3}$ (10.0), $\mathrm{MnCl}_{2} \cdot 4 \mathrm{H}_{2} \mathrm{O}$ (5.0), $\mathrm{CoCl}_{2} \cdot 6 \mathrm{H}_{2} \mathrm{O}$ (190.0), $\mathrm{ZnSO}_{4} \cdot 7 \mathrm{H}_{2} \mathrm{O}$ (144.0), $\mathrm{CuCl}_{2} \cdot 2 \mathrm{H}_{2} \mathrm{O}$ (10.0), $\mathrm{Na}_{2} \mathrm{MoO}_{4} \cdot 2 \mathrm{H}_{2} \mathrm{O}$ (36.0), $\mathrm{NiCl}_{2} \cdot 6 \mathrm{H}_{2} \mathrm{O}$ (24.0). Vitamins were filter-sterilized and added to final concentrations $(\mu \mathrm{g} / \mathrm{L})$ of 4-Aminobenzoic acid (4.0), D(+)-biotin (1.0), Nicotinic acid (10.0), D(+)-Pantothenic acid, calcium salt (5.0), Pyridoxine-HCl (15.0), Cyanocobalamin (5.0), and Thiamine$\mathrm{HCl}$ (10.0).

\section{Genomic DNA Extraction}

Genomic DNA was extracted as described by Chen et al. (2005). The quality and quantity of the extracted genomic DNA were checked by agarose gel electrophoresis and a NanoDrop spectrophotometer (Thermo Scientific).

\section{Genome Sequencing and Assembly}

Genomic DNA of $K$. profundi FT102 was sequenced using a nextgeneration sequencing platform. Illumina HiSeq $2000\left(2^{*} 120 \mathrm{bp}\right.$, $0.5-\mathrm{kb}$ insert size) sequencing was performed by BGI (Shenzhen, China), and de novo assembly was performed by using the Velvet assembler v.1.2.10 (Zerbino and Birney, 2008) with the following parameters: min kmer 31; max kmer 99; kmer step 6; and insert length 500. Eight contigs were assembled, including one containing an rRNA operon with doubled coverage and two short ones $(<550 \mathrm{bp}$ ). Then, these contigs were ordered according to the completed genome of K. koreensis DSM 16069. All gaps were filled using polymerase chain reaction (PCR) and Sanger sequencing (Supplementary Material S1). The coding sequence of $d n a A$ was set as the genomic start in the positive strand.

\section{Genomic Data Collection}

We collected five Kangiella (including K. profundi FT102) and 18 genomes of other Oceanospirillales (Table 1) from the NCBI database ${ }^{1}$. Both nucleotide and protein sequences were used in this study. The scaffold genome of K. aquimarina DSM 16071 was completed by PCR and sequencing in the present study.

\section{Genome Annotation and Comparative Analysis}

Replication origins (oriCs) in bacterial genomes were predicted using Ori-Finder ${ }^{2}$, which predicts based on analysis of the base

\footnotetext{
${ }^{1}$ http://ftp.ncbi.nlm.nih.gov/genomes

${ }^{2}$ http://tubic.tju.edu.cn/Ori-Finder/
} 
TABLE 1 | Genomic features of the Kangiella strains used in present study.

\begin{tabular}{|c|c|c|c|c|c|}
\hline Species name & $\begin{array}{l}\text { Kangiella } \\
\text { aquimarina } \\
\text { DSM } 16071\end{array}$ & $\begin{array}{l}\text { Kangiella } \\
\text { geojedonen } \\
\text { sis KCTC } 23420\end{array}$ & $\begin{array}{l}\text { Kangiella } \\
\text { koreensis } \\
\text { DSM } 16069\end{array}$ & $\begin{array}{l}\text { Kangiella } \\
\text { profundi } \\
\text { FT102 }\end{array}$ & $\begin{array}{l}\text { Kangiella } \\
\text { sediminilitoris } \\
\text { KCTC } 23892\end{array}$ \\
\hline Genome Size & $2,686,123$ & $2,495,242$ & $2,852,073$ & $2,653,010$ & $2,496,140$ \\
\hline$\% \mathrm{GC}$ & $43.72 \%$ & $43.78 \%$ & $43.69 \%$ & $43.81 \%$ & $43.40 \%$ \\
\hline tRNA operon & 41 & 41 & 41 & 41 & 41 \\
\hline rRNA number & 2 & 2 & 2 & 2 & 2 \\
\hline ORF number & 2446 & 2208 & 2632 & 2484 & 2308 \\
\hline ORF total length & $2,438,865$ & $2,244,783$ & $2,594,526$ & $2,382,009$ & $2,250,390$ \\
\hline \%ORF length & $90.79 \%$ & $89.96 \%$ & $90.97 \%$ & $89.79 \%$ & $90.15 \%$ \\
\hline SignalP & 317 & 298 & 339 & 305 & 283 \\
\hline$\%$ signalP & $12.96 \%$ & $13.50 \%$ & $12.88 \%$ & $12.28 \%$ & $12.26 \%$ \\
\hline TM & 606 & 533 & 701 & 610 & 581 \\
\hline$\% \mathrm{TM}$ & $24.78 \%$ & $24.14 \%$ & $26.63 \%$ & $24.56 \%$ & $25.17 \%$ \\
\hline No. of Paralogs & 130 & 121 & 150 & 142 & 133 \\
\hline ORF in paralogs & 344 & 296 & 396 & 370 & 332 \\
\hline
\end{tabular}

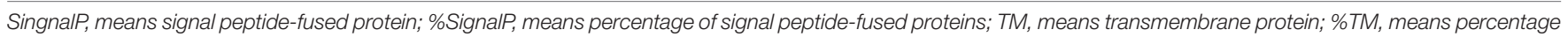
of transmembrane proteins.

composition asymmetry using the Z-curve method, distribution of DnaA boxes, and the occurrence of genes frequently close to oriCs (Gao and Zhang, 2008). Putative protein-coding sequences were predicted using Glimmer 3 (Delcher et al., 1999), which was trained with the CDS (coding sequences) from four completely sequenced Kangiella genomes. Manual curation of all coding sequences was performed by examining the database hits of BLAST 2.2.25+against the NR database and the RAST server (Aziz et al., 2008). tRNA and rRNA genes were predicted using tRNAscan-SE 1.3.1 (Lowe and Eddy, 1997) and RNAmmer 1.2 (Lagesen et al., 2007), respectively. Signal peptides of the ORFs were predicted with SignalP 4.1 (Petersen et al., 2011; Supplementary Material S3). Peptidase genes were predicted with MEROPS batch BLAST (Rawlings et al., 2016; Supplementary Material S3).

Protein families of 23 Oceanospirillales strains including 5 Kangiella were clustered using a local OrthoMCL 2.0.9 (Li et al., 2003) with the following cut-off values: identity, 30\%; coverage, 50\%; E-value, 1e-5; score, 40; and MCL Markov clustering inflation index, 1.5. To identify the Kangiella signature proteins, the core genome components were blasted using BLASTP against the non-redundant protein sequence (nr) database, excluding the Kangiella genus. A protein was considered a Kangiella signature protein if there were no BLAST hits with acceptable E-values $\left(<10^{-1}\right)$, similarity $(>20 \%)$, or coverage $(>50 \%)$. GO (gene ontology) term enrichment was performed using Blast2GO PRO 3.0. COG (cluster of orthologous genes) classification was performed using the WebMGA online server ${ }^{3}$.

\section{Metabolic Network of Kangiella}

The core metabolic network of Kangiella was constructed based on the core genome information of the five Kangiella strains using KEGG BlastKOALA ${ }^{4}$.

${ }^{3} \mathrm{http}: / /$ weizhong-lab.ucsd.edu/metagenomic-analysis/server/cog/

${ }^{4}$ http://www.kegg.jp/blastkoala/

\section{Genome Rearrangement Analysis}

Locally collinear blocks (LCBs) of the five Kangiella strains were investigated using Mauve aligner 2.4.0 (Darling et al., 2004). Each LCB represents a region of homologous sequence without rearrangement among genomes.

\section{GC-Skew Analysis}

GenSkew online application ${ }^{5}$ was used to compute and plot GCskew data.

\section{RESULTS}

\section{Genomic Features of Kangiella profundi FT102}

The complete genome sequence of $K$. profundi FT102 is composed of a circular chromosome of 2,653,010 bp with $43.81 \%$ GC content (Figure 1). The coding region covers $89.79 \%$ of the genome, encoding 2,484 proteins, of which 2,042 proteins were annotated with COG classification (Supplementary Table S1). Translation, ribosomal structure, general function prediction only (R), biogenesis (J), and amino acid transport, and metabolism (E) were the most abundant COG categories (7.81, 6.60 , and $6.42 \%$, respectively). The genome also encodes 41 tRNAs, as well as 2 rRNA operons. Signal peptides and transmembrane helices account for $12.28 \%$ (305) and $24.56 \%$ (610) of the protein-coding genes, respectively (Table 1 ).

Moreover, eight genomic islands (GIs) were predicted in the K. profundi FT102 strain (Figure 1). One of the GIs encodes 15 proteins (orf01390 to orf01530), including a putative type VI secretion system and a related lysozyme, which might provide antibacterial ability to $K$. profundi FT102. Another GI with the least GC content compared to the rest of the genome was

${ }^{5}$ http://genskew.csb.univie.ac.at/ 


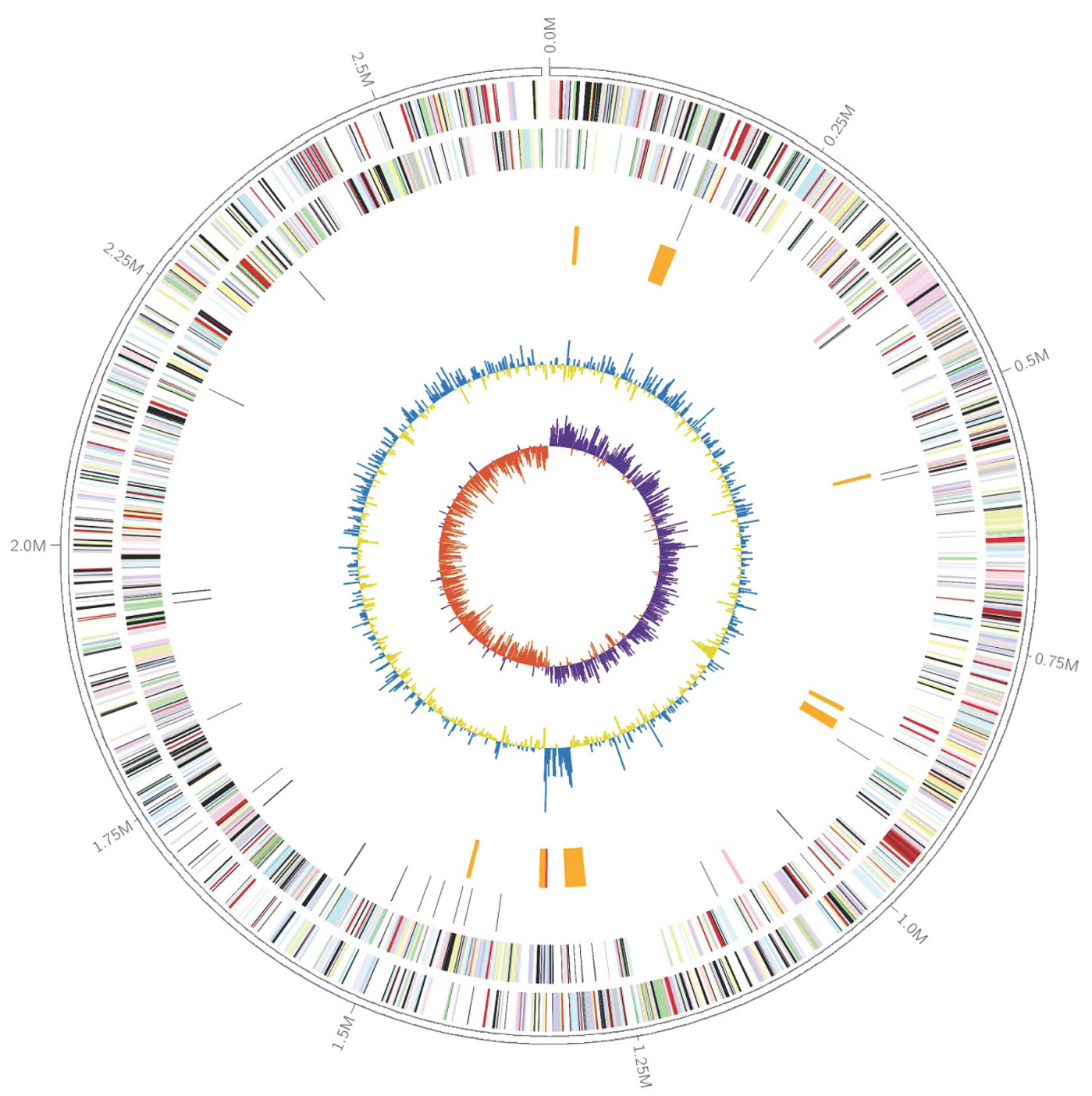

FIGURE 1 | Graphical representation of the Kangiella profundi FT102 genome. Genes on the forward (shown in Outer circle) and reverse (shown in Inner circle) strands are colored according to their cluster of orthologous genes (COG) categories, RNA genes are highlighted with different colors (tRNAs orange, rRNAs red, other RNAs green), gene islands and GC content are shown in black, and GC skew is shown in light green/orange.

predicted to harbor gene clusters for the biosynthesis of capsular polysaccharides. In contrast, the region with the highest GC content, located next to the GC-skew, was annotated as a putative heavy-metal resistance island against $\mathrm{Zn} / \mathrm{Co} / \mathrm{Cd}$.

\section{Signature Proteins of the Genus Kangiella}

The Kangiella genus is of phylogenetic interest because of its very isolated location in Oceanospirillales. K. koreensis is the first type species of this genus to be described and was selected for whole-genome sequencing as part of the Genomic Encyclopedia of Bacteria and Archaea project (Han et al., 2009). With five complete genomes at hand, we identified the signature proteins of Kangiella by BLAST analysis on the core genome with the following cutoff: at least $60 \%$ identity (e-value: $1 \mathrm{e}-30)$ with each other in the same orthologous families and less than 30\% identity (e-value: 1e-1) with any non-Kangiella hits from the NR database. Under the cutoff, four orthologous families (KangOF24, 566, 656, and 804) appeared as Kangiella specific and were annotated as hypothetical proteins. Notably, two copies of KangOF24 were observed in K. sediminilitoris KCTC 23892 (AOE50183.1 and AOE50181.1). These signature proteins could be used as genusspecific molecular markers to identify Kangiella independently of detection of $16 \mathrm{~S}$ rRNA gene as a phylogenic marker.

\section{Analysis of Orthologous Gene Families}

The genomic features of the five Kangiella species used in the present study are shown in Table 1. The Markov clustering algorithm OrthoMCL was used to identify orthologous gene families. Further analysis of these families in the five Kangiella genomes revealed 3,544 orthologs (Figure 2 


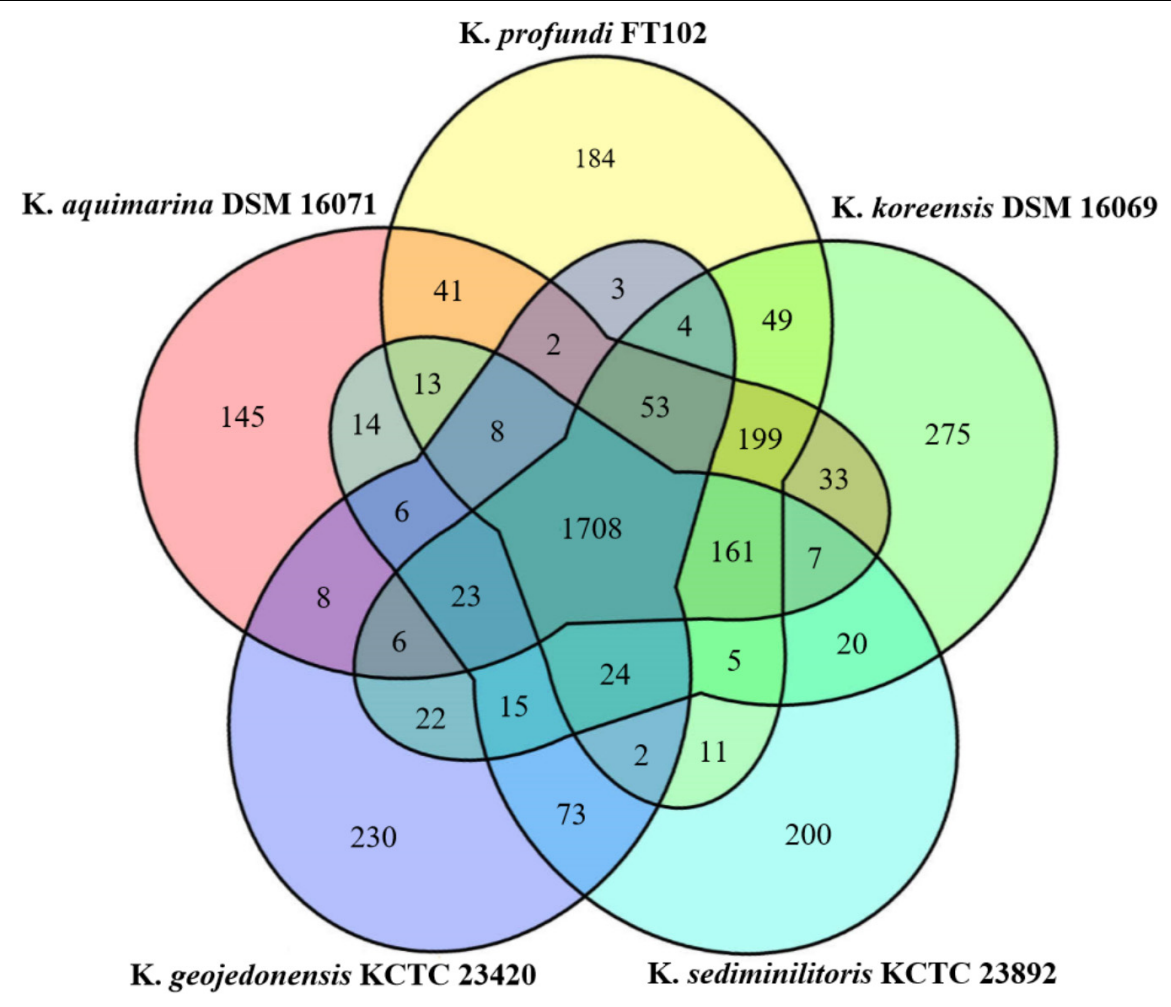

FIGURE 2 | Venn diagram of the orthologous families of the five Kangiella species. The size of the core genome is shown in the center, while the numbers of strain-specific genes in Kangiella strains are shown in the petals.

and Supplementary Table S2), which includes (1) 1,708 core genome components, of which only 21 orthologs contain duplication in at least one genome (Supplementary Table S3); (2) 802 components in the dispensable genome, which have representatives in at least two but not all of the five genomes; and (3) 1,034 components that were uniquely present only in one genome, which include only 10 lineage-unique (LSE) gene families and 1,024 singletons. According to the COG classification (Supplementary Figure S1 and Supplementary Table S4), it is evident that core genes mainly belong to categories J (translation, ribosome structure, and biogenesis), C (energy production and conversion), D (cell cycle control, cell division, and chromosome partitioning), F (nucleotide transport and metabolism), G (carbohydrate transport and metabolism), $\mathrm{H}$ (coenzyme transport and metabolism), I (lipid transport and metabolism), and $\mathrm{L}$ (replication, recombination, and repair); the unique genes are involved in $\mathrm{V}$ (defense mechanisms); and dispensable genes are found more often associated with $\mathrm{P}$ (inorganic ion transport and metabolism), and $\mathrm{T}$ (signal transduction mechanisms).

\section{Strain-Specific Losses and Gains in the Metabolic Pathways in Kangiella}

To determine the differences in the metabolic potential among Kangiella strains, we compared the GO terms from each strain, and strain-specific gene gains and losses were identified. To avoid the biases of annotation, "gained" GO terms were derived from strain-specific orthologous families that were not possessed by any of the other four strains. On the other hand, "lost" GO terms were retrieved only from those dispensable orthologous families that were owned by each of the four strains and missing in only one. Genes related to these GO terms were manually checked using both KEGG pathways and BLASTP analysis.

The results suggest that $K$. profundi FT102 gained a type III restriction endonuclease (orf15860) and a related DNA methyltransferase (orf15870), as well as a type VI secretion system. The gene clusters related to sulfide reduction and arginine biosynthesis were missing in the FT102 strain; this absence was also validated through PCR and sequencing (Supplementary Figure S2 and Supplementary Material S2).

Kangiella aquimarina DSM 16071 was found to be the only strain with a type I restriction-modification system, including site-specific deoxyribonuclease activity (WP_033414025.1) and related methyltransferase (WP_033414024.1). Moreover, 2isopropylmalate synthases were observed in other analyzed strains but were absent in K. aquimarina DSM 16071. 2isopropylmalate is an important substrate for the biosynthesis of leucine from pyruvate and could be catalyzed to leucine along with acetyl-CoA, 3-methyl-2-oxobutanoate, and water. However, K. aquimarina DSM 16071 contains isopropylmalate isomerase (WP_018624573.1), which could transform (2R,3S)3 -isopropylmalate to (2S)-2-isopropylmalate. Therefore, it was suggested that $K$. aquimarina DSM 16071 could synthesize leucine, similar to other species, using an alternate pathway. 
Kangiella koreensis DSM 16069 represents the largest genome size compared to the other four strains used in this study, and no strain-specific GO term loss was found in K. koreensis DSM 16069. Furthermore, K. koreensis DSM 16069 was found to exhibit putative nitrate reduction capability. A gene cluster with at least 19 proteins (ACV26744.1-ACV26762.1) and 12 GO terms related to Mo-molybdopterin cofactor biosynthesis and dissimilatory nitrate reduction were found limited to K. koreensis DSM 16069.

Although, the genome size of K. geojedonensis KCTC 23420 is the smallest among all the strains used herein, it was the only one predicted to have a putative capacity for synthesizing all 20 amino acids. A gene cluster (AKE51219.1-AKE51221.1) related to branched-chain amino acid biosynthetic and metabolic processes was found in K. geojedonensis KCTC 23420, while it was missing in the rest of the four Kangiella strains. Furthermore, the presence of homoserine kinase (AKE52760.1) possibly represents a strain-specific biosynthetic pathway from L-aspartate to threonine via O-phospho-L-serine in K. geojedonensis KCTC 23420. Additionally, 10 strain-specific GO terms in 6 orthologous families were found in the second island of $K$. geojedonensis KCTC 23420 (consisting of 21 protein-coding genes) and are involved in capsule biosynthesis. However, they lack genes involved in bacterial respiration (such as cytochrome c oxidase subunits and HemN), oxidative resistance (such as superoxide dismutase), heavy metal transport, and nitrite utilization.

Kangiella sediminilitoris KCTC 23892 was the only strain with an ammonium transmembrane transporter (AOE50237.1), while it was also the only one among the five Kangiella strains that lacked aspartate-ammonia ligase, whose function could be supplemented by asparagine synthase (AOE49243.1).

\section{Reconstruction of the Basic Metabolic Pathways of Carbohydrate and Amino Acid}

Based on the comparison KEGG pathway analysis described above, the main metabolic network of the Kangiella strains were reconstructed. The glycolysis, tricarboxylic acid (TCA) cycle, and amino acid biosynthesis and transport pathways are shown in Figure 3. Although Aliikangiella marina GYP- $15^{T}$ was reported as a carbohydrate utilizer (Wang et al., 2015), the gene encoding carbohydrate transporter or glucose kinase was not predicted in the Kangiella strains, which might be the reason that the heterotrophic growth of Kangiella strains could not be supported when using glucose as the sole carbon source (data not shown). Therefore, it is possible that carbohydrates are derived from central carbon metabolism (such as pyruvate) and transformed via reverse glycolysis and the pentose phosphate pathway. Fatty acids could be one of the carbon sources, as the genes coding for the key enzymes in the fatty acid beta-oxidation pathway could be found in the genomes of Kangiella. Amino acids could also feed central carbon metabolism via deamination and/or transamination, and most of them could be synthesized by each strain.

Diversity in metabolic capability in the biosynthesis of certain amino acids, including valine, isoleucine, arginine, and phenylalanine, was observed among the analyzed strains (Figure 3). K. geojedonensis KCTC 23420 appeared to be able to synthesize valine and isoleucine, while $K$. profundi FT102 could not synthesize arginine. Additionally, biosynthesis of phenylalanine was found to be missing in both $K$. profundi FT102 and K. sediminilitoris KCTC 23892 because of the loss of a gene cluster containing phospho-2-dehydro3-deoxyheptonate aldolase and prephenate dehydratase (Supplementary Figure S2). Particularly, the predicted disability of the phenylalanine and arginine biosynthesis in $K$. profundi FT102, were experimentally validated by growth curve analysis under various defined media with amino acids as the sole carbon and nitrogen source (Figure 4). Interestingly, we detected various kinds of secretory peptidases, which are exported by TIISS/Tat/Sac system to degrade extracellular proteins into amino acids and peptides in these strains. Furthermore, seven types of amino acid/peptide transporters were also identified, which could support the intake of these amino acids/peptides from the extracellular environment.

\section{Genome Reduction and Metabolic Simplicity of Kangiella Strains}

The genomic size of the Kangiella strains is smaller than that of any other free-living Oceanospirillales strains. To gain insights into the genome reduction in Kangiella, we performed comparative genome analysis with 18 non-Kangiella completely sequenced genomes of Oceanospirillales strains (Supplementary Table S5). We particularly focused on the investigating genomic redundancy, functional gene categories and genome function reduction. OrthoMCL clustering results showed that each Kangiella strain possesses at most 150 paralogous gene families within 396 genes, whereas non-Kangiella strains exhibit at least 351 paralogous families within 1,118 genes, demonstrating that low genomic redundancy is the primary reason behind the genome reduction. There were fewer COG categories in Kangiella strains than in their non-Kangiella counterparts (Supplementary Table S1), especially for carbohydrate transport and metabolism (COG category $\mathrm{G}$ ), genes in the mobilome (COG category $\mathrm{X}$ ) and cell motility (COG category N). Each Kangiella strain exhibits no more than 41 COG category G orthologous proteins, while non-Kangiella strains have at least 60 COG category G, demonstrating that Kangiella has a decreased preference for carbohydrate transport and metabolism. On the average, Kangiella strains have only four COG category X orthologous proteins, while non-Kangiella strains have 48, which reflects less horizontal gene transfer during the evolution of Kangiella. There also seemed to be fewer COG category N orthologous proteins than the average in non-Kangiella strains, which might be mainly because Kangiella strains lack flagellum (Figure 5).

To gain insights into the reduction in Kangiella-specific function, we clustered all five Kangiella protein sequences together with their 18 counterparts using OrthoMCL. Among 23,058 Oceanospirillales orthologous families (OceaOFs) (Supplementary Table S6), we found 96 families that were only absent in Kangiella and called them "Kangiella-specificabsences." Genes both from these families and all five Kangiella 


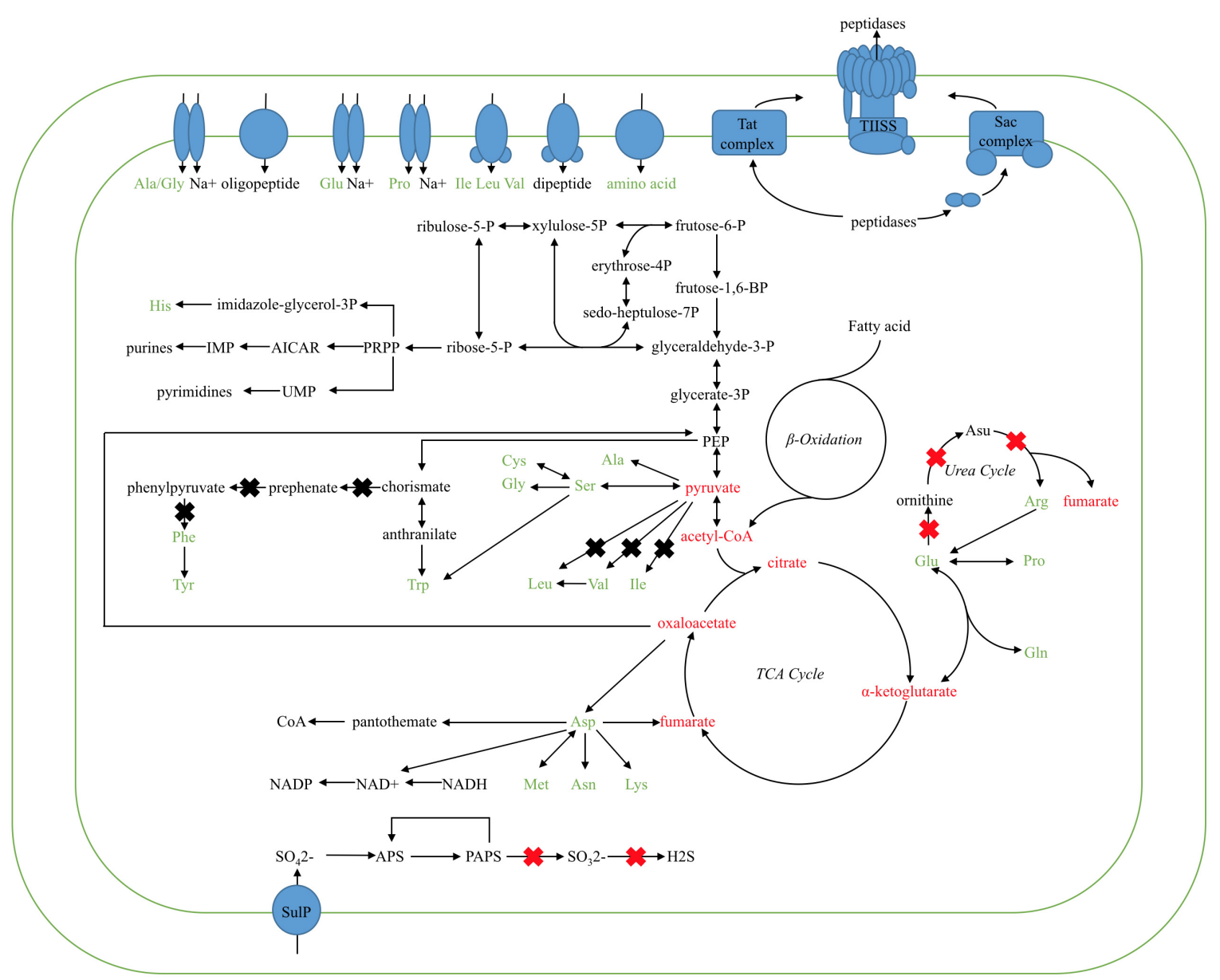

FIGURE 3 | Proposed metabolic pathways of the TCA cycle, inferred from the Kangiella core genome. Ovals in blue represent the amino acid/oligopeptide transporters and protein-secreting systems. Black arrows show the flow of the metabolic pathway. X marks in the red color show the genes absent only in K. profundi FT102 (confirmed by PCR and growth experiments), whereas X marks in black represent the genes absent in K. profundi FT102 and at least one other species (but not all the species used here).

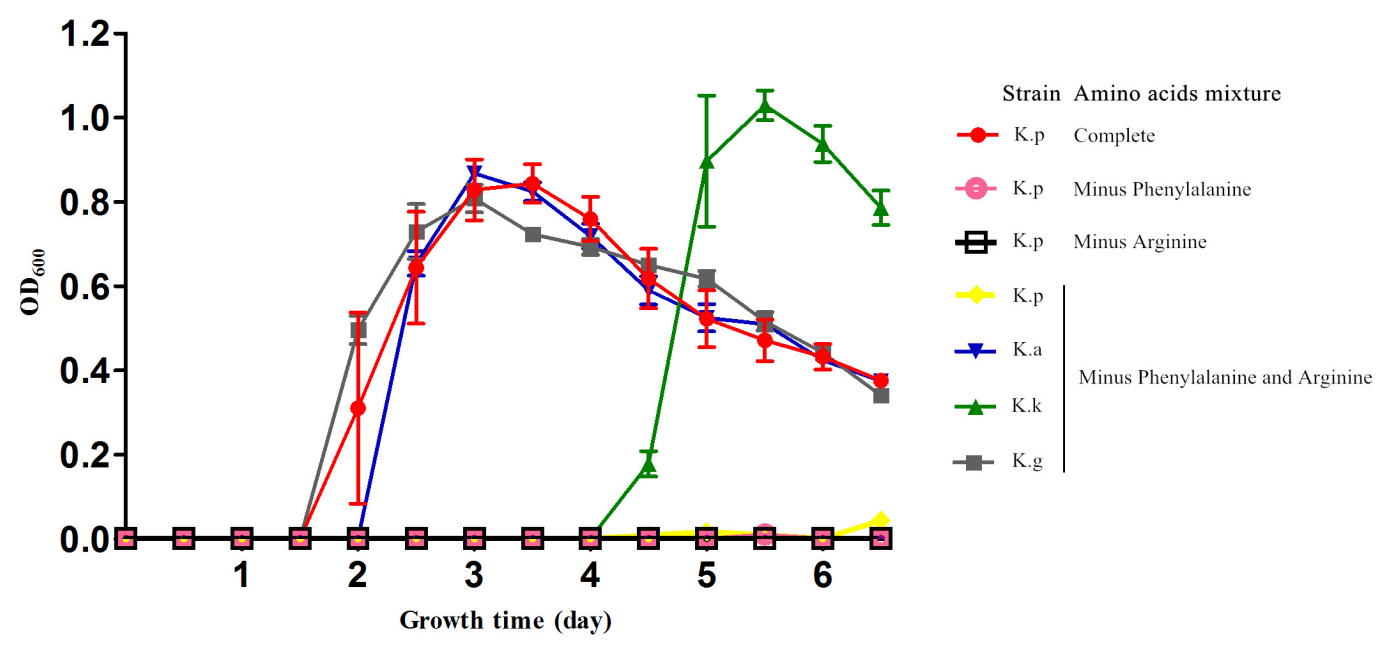

FIGURE 4 | Growth curve of the four Kangiella strains cultured in the defined amino acids medium. K.p, Kangiella aquimarina DSM 16071; K.k, Kangiella koreensis DSM 16069; K.g, Kangiella geojedonensis KCTC 23420; and K.p, Kangiella profundi FT102. Complete: 20 amino acids; Minus Phenylalanine: phenylalanine-absent 19 amino acids; Minus Arginine: arginine-absent 19 amino acids; Minus Phenylalanine and Arginine: phenylalanine- and arginine-absent 18 amino acids. 


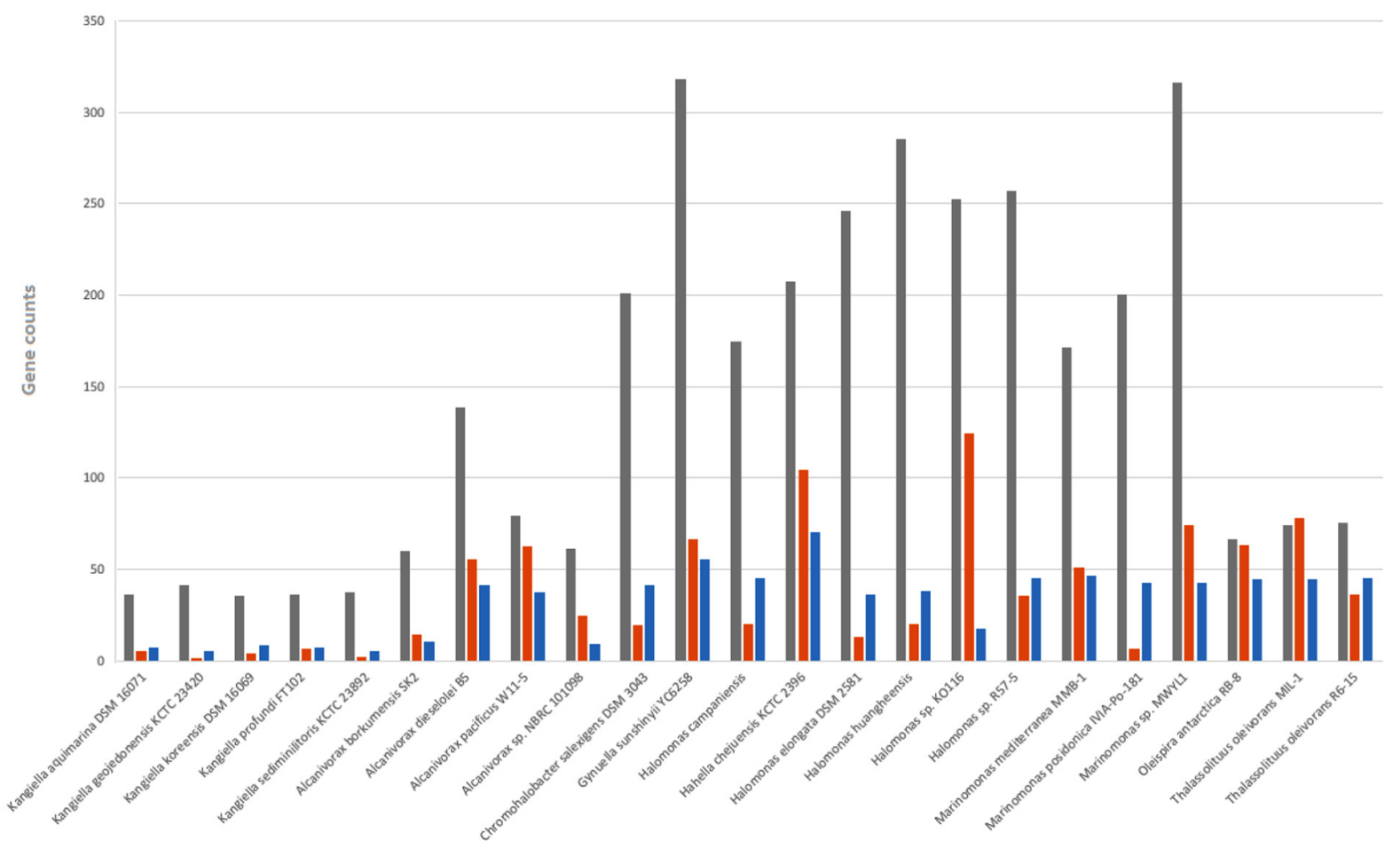

FIGURE 5 | Number of genes belonging to selected COG categories in all 23 strains in Oceanospirillales. G shown in gray is related to carbohydrate transport and metabolism; $\mathrm{N}$ shown in orange is related to cell motility; and $\mathrm{X}$ shown in blue is related to mobility.

genomes were annotated against the GO database. If a GO term from these "Kangiella-specific-absent" families was also found to be absent in all the Kangiella genomes, this pathway was inferred to be lost in Kangiella. Approximately 31 GO terms from 24 OceaOFs were found missing in Kangiella only (Supplementary Table S7). All the non-Kangiella Oceanospirillales were found to contain a phosphoenolpyruvate-dependent sugar phosphotransferase system; in contrast, this protein was missing in Kangiella. Glycerol is the primary carbon source available to halophilic heterotrophic communities. Glycerol kinase (EC 2.7.1.30; ATP-glycerol 3-phosphotransferase) is required for glycerol metabolism, and Kangiella strains were found to be deprived of it, suggesting that glycerol is not the carbon source in Kangiella. Malate synthase works together with isocitrate lyase in the glyoxylate cycle to bypass two oxidative steps of the Krebs cycle and permit carbon incorporation from acetate or fatty acids in many microorganisms. Kangiella is also lacking these enzymes, which demonstrates the inertness of gluconeogenesis in Kangiella. Phosphoenolpyruvate carboxylase was also missing in Kangiella. In the aspect of amino acid biosynthesis and metabolism, the glutamate synthase, L-alanine:2-oxoglutarate aminotransferase and threonine dehydratase are missing in all Kangiella genomes. Kangiella strains also lack GO:0008942 (F:nitrite reductase [NAD(P)H] activity) and GO:0042128 (P:nitrate assimilation), which suggests that Kangiella cannot perform assimilatory nitrate reduction. Many halophilic microorganisms accumulate ectoine (1,4,5,6-tetrahydro-2-methyl-4-pyrimidine carboxylic acid) to counteract heat, cold, desiccation, and high salinity. The operon with L-2,4-diaminobutyric acid acetyltransferase, 4-aminobutyrate aminotransferase, and ectoine synthase is missing in Kangiella.

\section{High Extracellular-Protein/Peptide Degradation Potential of Kangiella}

The low genomic redundancy in Kangiella, was observed in the present study (Supplementary Table S3). Surprisingly, only $21(1.23 \%)$ orthologs with duplications in the core genome and five of them (KangOF4, 8, 9, 15, and 19) were annotated to be SignalP-fused peptidases (SignalP) that involved in proteolysis (GO:0006508). Our experimental results also demonstrated that Kangiella strains could grow solely on amino acids as the carbon and nitrogen source (Figure 4). In addition, the biomass of Kangiella was increased if tryptone in marine broth 2216 medium has been doubled to make a enriched marine peptone medium (Figure 6). Therefore, we proposed that extracellular protein degradation and amino acid utilization are essential and prominent features of Kangiella.

Although the genome sizes of Kangiella strains were smaller than those of the other genus in Oceanospirillales, the percentage of SignalP-fused peptidases in Kangiella strains (1.68-2.36\%) was significantly higher than that in the other genomes belonging to the order Oceanospirillales (0.25-0.80\%) (Table 2 and Supplementary Table S8). Furthermore, Kangiella strains also exhibit diverse types of peptidases. Approximately 67-71 


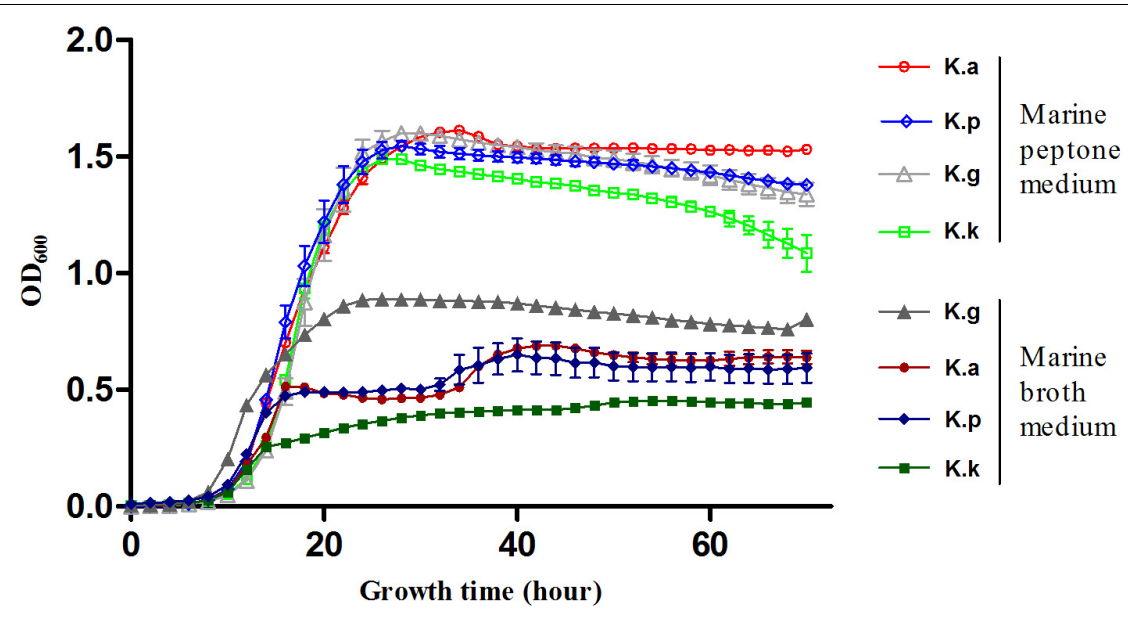

FIGURE 6 | Growth curve of four Kangiella strains cultured in marine peptone medium grouped in "Marine peptone medium" and marine broth 2216 grouped in "Marine broth medium." K.a. K. aquimarina DSM 16071; K.K. K. Koreensis DSM 16069; K.g, K. geojedonensis KCTC 23420 ; and K.p, K. profundi FT102.

TABLE 2 | Comparison of peptidases in all the 23 genomes used in this study.

\begin{tabular}{|c|c|c|c|c|}
\hline Sr. No. & Strain name & $\begin{array}{l}\text { Total No. of } \\
\text { Peptidase }\end{array}$ & $\begin{array}{l}\text { Secretory } \\
\text { peptidase }\end{array}$ & $\begin{array}{l}\text { No. of Secretory } \\
\text { Peptidase/MB }\end{array}$ \\
\hline 1 & Kangiella aquimarina DSM 16071 & 142 & 56 & $20.85^{*}$ \\
\hline 2 & Kangiella geojedonensis KCTC 23420 & 140 & 50 & $20.04^{*}$ \\
\hline 3 & Kangiella koreensis DSM 16069 & 156 & 62 & $21.74^{*}$ \\
\hline 4 & Kangiella profundi FT102 & 142 & 54 & $20.40^{*}$ \\
\hline 5 & Kangiella sediminilitoris KCTC 23892 & 142 & 43 & $17.23^{*}$ \\
\hline 6 & Alcanivorax borkumensis SK2 & 108 & 20 & 6.41 \\
\hline 7 & Alcanivorax dieselolei B5 & 151 & 21 & 4.26 \\
\hline 8 & Alcanivorax pacificus W11-5 & 121 & 20 & 4.80 \\
\hline 9 & Alcanivorax sp. NBRC 101098 & 107 & 21 & 6.78 \\
\hline 10 & Chromohalobacter salexigens DSM 3043 & 123 & 15 & 4.06 \\
\hline 11 & Gynuella sunshinyii YC6258 & 156 & 29 & 4.48 \\
\hline 12 & Halomonas campaniensis & 133 & 25 & 3.46 \\
\hline 13 & Hahella chejuensis KCTC 2396 & 219 & 54 & 13.25 \\
\hline 14 & Halomonas elongata DSM 2581 & 122 & 12 & 2.95 \\
\hline 15 & Halomonas huangheensis & 147 & 23 & 4.83 \\
\hline 16 & Halomonas sp. KO116 & 132 & 21 & 4.06 \\
\hline 17 & Halomonas sp. R57-5 & 141 & 20 & 3.97 \\
\hline 18 & Marinomonas mediterranea MMB-1 & 120 & 15 & 3.20 \\
\hline 19 & Marinomonas posidonica IVIA-Po-181 & 111 & 15 & 3.85 \\
\hline 20 & Marinomonas sp. MWYL1 & 122 & 11 & 2.16 \\
\hline 21 & Oleispira antarctica RB-8 & 119 & 15 & 3.40 \\
\hline 22 & Thalassolituus oleivorans MIL-1 & 117 & 20 & 5.10 \\
\hline 23 & Thalassolituus oleivorans R6-15 & 115 & 22 & 5.84 \\
\hline
\end{tabular}

"*" means $p$-value $<0.01$.

peptidase subfamilies were predicted in Kangiella, of which 26 subfamilies were signal-fused peptidases, whereas only 55 subfamilies were identified in their non-Kangiella counterparts on average, and only 14 were signal-fused peptidases. Moreover, eight families of peptidases with SignalP (S8A, S9B, S9C, S41A, M19, M16B, and M38) were found to be remarkably abundant in Kangiella. We also identified three Kangiella-specific SignalPfused protease families (S10, S46, and M28D).
S8A peptidases are the most abundant peptidase superfamily in Kangiella genomes. A gene cluster containing several tandem S8A superfamily peptidases (Supplementary Figure S3), including two orthologs (KangOF8 and 9) having multiple copies in the core genome was also identified in the Kangiella strains. KangOF9, which was annotated as a cold-active alkaline serine protease, was found with up to three copies per Kangiella genome. Interestingly, the paralogs of these proteases in KangOF9 showed 
overall high sequence identity, except that an additional PKD domain was observed in each of the longer ones.

\section{DISCUSSION}

Genomic reduction is a significant characteristic of Kangiella among Oceanospirillales. It was reported that both loss of entire gene families and deletion of paralogs within multigene families could contribute to genome size reduction in marine bacteria (Lerat et al., 2005), and the smaller genome size of the marine cyanobacterial genus Prochlorococcus resulted from gene loss compared with its closely related genus Synechococcus (Luo et al., 2011). Our results showed a similar mechanism of genomic reduction, including de-redundancy and genus-specific loss of orthologs, in genus Kangiella compared to other nonKangiellaceae Oceanospirillales.

It should be noted that in the background of global genomic reduction and de-redundancy of Kangiella, genomic plasticity and expansion also exist. Multigenome alignments (Figure 7) revealed that in the central position near the GC-skew reversion points of each Kangiella genome, there is an aberrant GC content genomic region $(0.05-0.2 \mathrm{Mb})$ with poor colinearity. Such "hot block" for insertion and recombination was found to contain a lot of heavy metal and arsenic resistance genes in all Kangiella genomes, except K. geojedonensis KCTC 23420.

In the aspect of central metabolism, we predicted and experimentally confirmed that different Kangiella strains showed different defects in the capabilities of amino acid biosynthesis. For example, K. profundi FT102 lacks biosynthetic pathways of isoleucine, valine and phenylalanine and arginine, while $K$. koreensis DSM 16069 and K. aquimarina DSM 16071 only lack the first two. Interestingly, the phylogenetic tree based on $16 \mathrm{~S}$ rRNA gene sequences showed that K. aquimarina DSM 16071 and $K$. profundi TF102 are more closely related than K. koreensis DSM 16069 (Choe et al., 2015). However, K. aquimarina DSM 16071 and K. koreensis DSM 16069 from the same sampling location showed the same capability of amino acids biosynthesis.

Furthermore, the subunits of sulfite reductase were strain-specifically missing in $K$. profundi FT102 (Supplementary Figure S2) as mentioned above, and we did not identify any functionally complementary gene in its genome.

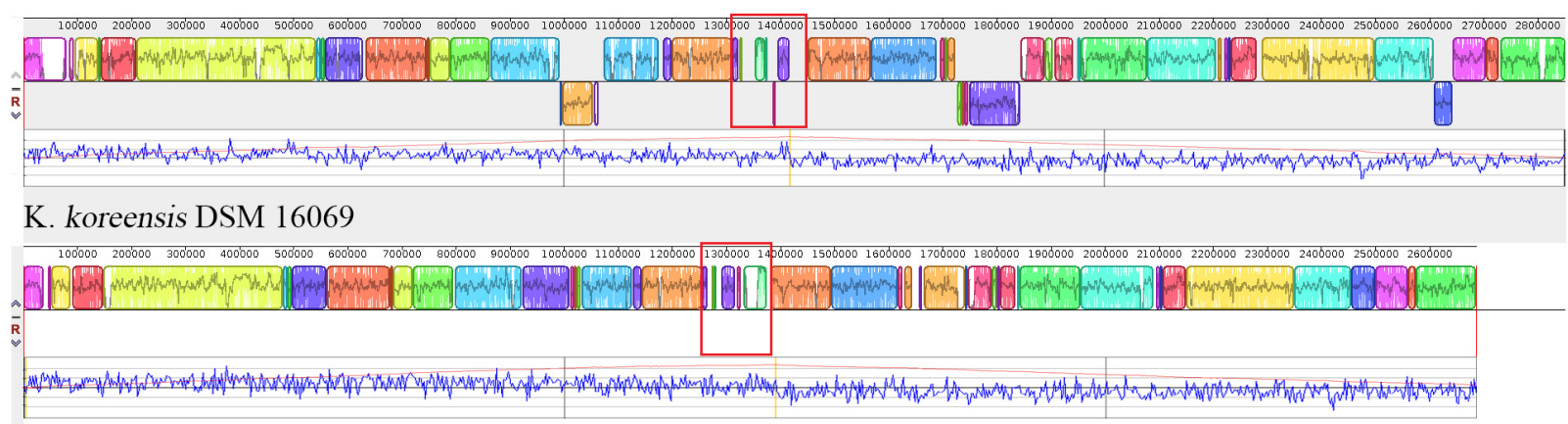

K. aquimarina DSM 16071

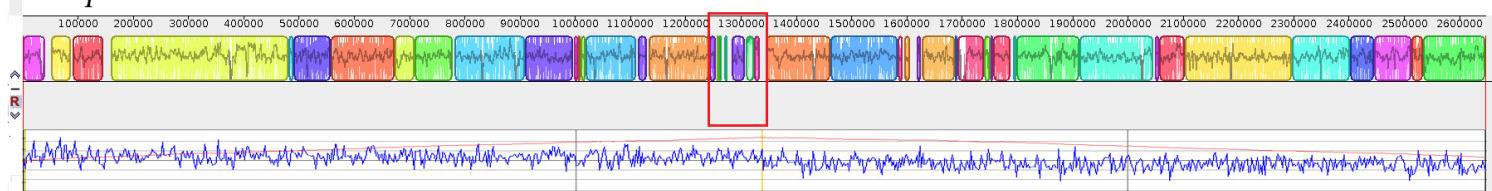

K. profundi FT102

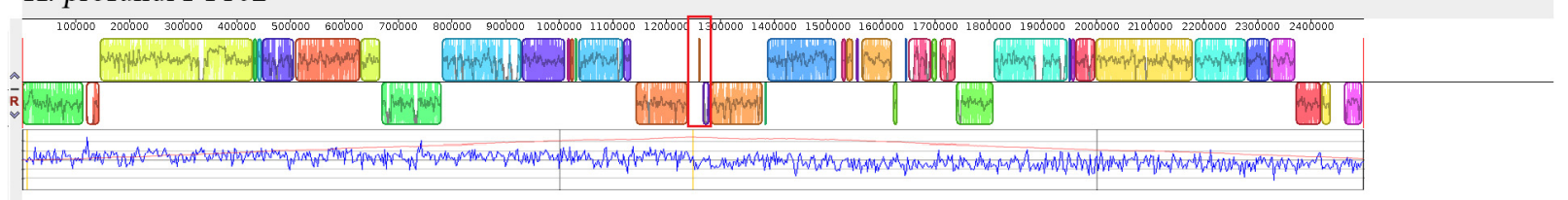

K. sediminilitoris KCTC 23892

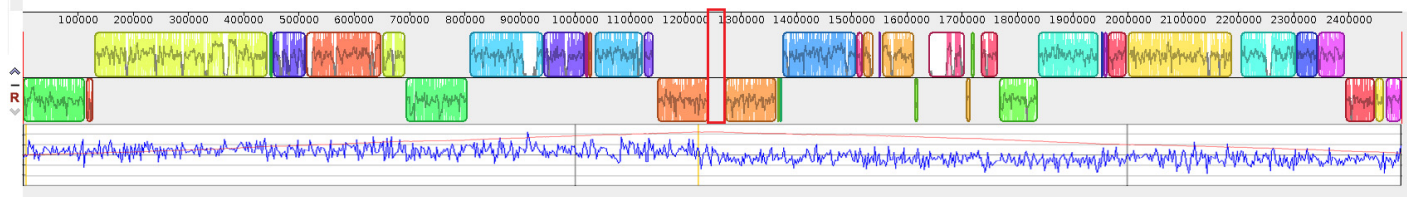

K. geojedonensis KCTC 23420

FIGURE 7 | Collinearity of the Kangiella genomes. Highlighted sites in red show the highly variable region of each genome. Blocks in the different colors represent different locally collinear blocks (LCBs). Local GC-skew (blue) and cumulative GC-skew (red) were plotted below LCBs, and the peak position of the cumulative GC-skew was highlighted in orange. 
Actually, the sediment samples used to isolate $K$. profundi FT102 were collected from the southwest Indian InterRidge (Xu et al., 2015), which contain a large amount of sulfides and iron hydroxides (Tao et al., 2011, 2012). As long as the demand of sulfide has been satisfied from the environment, loss of those related genes seems to have no detrimental effect in $K$. profundi FT102.

In the aspect of proteolysis, our previous study confirmed the extracellular protease activities of four Kangiella strains (K. profundi FT102, K. koreensis DSM 16069, K. aquimarina DSM 16071, and K. geojedonensis KCTC 23420). In general, a positive correlation of the biomass and the extracellular protease activities was found when these strains were cultured in marine broth 2216 (Xu et al., 2018).

It is also worth mentioning that unlike Aliikangiella marina GYP- $15^{T}$, which represents another genus in Kangiellaceae, and was confirmed to utilize carbohydrates (Wang et al., 2015), Kangiella could not grow in defined media using glucose as the sole carbon source (data not shown). Taken together, proteolysis of extracellular proteins rather than carbohydrate utilization plays an important role in the life style of Kangiella.

Peptidase constitute a very large and complex group of enzymes that differ in properties such as substrate specificity, active site and catalytic mechanism, $\mathrm{pH}$ and temperature optima, and stability profiles (Jisha et al., 2013). In the Kangiella strains, there are at least 43 extracellular peptidases that could be classified into 26 subfamilies. Most of them are non-redundant, indicating wide substrates specificity of peptidases in Kangiella. Besides, among the merely 21 gene families with redundancy in core genome of Kangiella, five are secreted peptidases. In detail, four (KangOF4, 8, 9 and 15) of them belong to S8 peptidase family, and KangOF19 belongs to M1 peptidase family.

The S8A family peptidases are the most abundant in Kangiella genomes, including the tandem gene cluster constituted with the genes of KangOF190, 9 and 8 (Supplementary Figure S3). S8 peptidase is the second largest family of characterized serine peptidases, and they have catalytic triad consisted of aspartate, histidine and serine (Yamagata et al., 1994), which differs from that S1, S9 and S10 serine peptidases families. S8 peptidase family could be divided into two subfamilies, i.e., subtilisin (S8A) and kexin (S8B) according to MEROPS peptidase database. The S8 peptidases are mostly secreted and non-specific peptidases, and probably involved in uptaking of nutrition, which could support the obligate proteolytic lifestyle of Kangiella.

Moreover, S9 family serine peptidases are also abundant in Kangiella (Supplementary Table S8). However, no redundancy of S9 family was found in the core genome of Kangiella. Different from S8 peptidases, most members of the S9 peptidases family show strict substrate specificity (Rawlings et al., 1991). The tendency to be streamline in the genome of Kangiella was evident in keeping many heterogeneous genes of S9 family members instead of gene duplication.

More interestingly, the S10 peptidase family members were only identified and are conserved in Kanagiella genomes compared to other genus in Oceanospirillales. Different from most other serine peptidase families (S53 being the exception), the $\mathrm{S} 10$ peptidases are only active at acidic $\mathrm{pH}$, e.g., carboxypeptidase Y with max activity at pH 5.5 (Jung et al., 1998). As the optimal pH for the growth of Kangiella is 7.0-8.0, the biological function of the putative S10 peptidases in Kangiella is still unclear. Quantitative analysis of the expression level of S10 peptidases in Kangiella challenged by different $\mathrm{pH}$ are still needed.

In the view of evolution, it is also interesting that the duplications of the peptidase families are still obvious in the Kangiella genomes under the background of genomic deredundancy. We proposed that such gene duplications are partly driven by the obligate carbon source acquisition strategy of Kangiella. However, K. sediminilitoris KCTC 23892 was an exception, which showed no abovementioned duplication of KangOF8 or KangOF9 (Supplementary Figure S3), as well as carriers the fewest SignalP-fused peptidases (only 69$86 \%$ of other stains) (Table 2 ). The reason might be that the duplication is not only driven by carbon acquisition but also by nitrogen acquisition. Actually, a strain-specific gene coding ammonia transporter could be only identified in $K$. sediminilitoris as mentioned above. Absorbing inorganic nitrogen from the environment might alleviate its dependency on protein-derived ammonia, thus alleviating the selective pressure that drives the multiplication of extracellular peptidase genes.

\section{CONCLUSION}

In the present study, the complete genome of the novel strain Kangiella profundi FT102 was sequenced, and gaps in the scaffold genome of $K$. aquimarina DSM 16071 were filled using PCR. Five sequenced Kangiella genomes, including the abovementioned two, were utilized along with 18 non-Kangiella genomes in a comparative genomics approach to explore the metabolic capabilities of Kangiella and gain insight into genome reduction in Kangiella. We reported here that low genomic redundancy and Kangiella-lineage-specific gene loss are two key factors behind genome reduction in Kangiella. Furthermore, a highly enriched extracellular protein degradation system compared to that of any other non-Kangiella species was identified in Kangiella. Despite the low genome redundancy in the core genome (only 21 paralogs), five of these paralogs were signal-fused proteases. The absence of a complete pathway for carbohydrate metabolism led to the belief that proteases are not only abundant but also essential for Kangiella survival, demonstrating the extraordinary protein degradation capabilities of Kangiella.

\section{AUTHOR CONTRIBUTIONS}

JX and JW designed the experiments and analysis. JW and MN performed the computational analysis. YL conducted the experiments. JW, YL, and JX wrote the manuscript, in consultation with all other authors. 


\section{FUNDING}

This study was supported by the National Basic Research Program of China ("973" Program 2014CB441503) and the National Natural Science Foundation of China (41676121 and 41376137).

\section{SUPPLEMENTARY MATERIAL}

The Supplementary Material for this article can be found online at: https://www.frontiersin.org/articles/10.3389/fmicb. 2018.01224/full\#supplementary-material

FIGURE S1 | Percentage of the genes belonging to different cluster of orthologous genes (COG) categories in the orthologous gene families in pangenome of the five Kangiella strains. Genes belonging to the core genome, that are dispensable and that are specific to species are shown in blue, orange and gray, respectively. A: RNA processing and modification; C: Energy production and conversion; D: Cell cycle control, cell division, chromosome partitioning; E: Amino acid transport and metabolism; F: Nucleotide transport and metabolism; G: Carbohydrate transport and metabolism; H: Coenzyme transport and metabolism; I: Lipid transport and metabolism; J: Translation, ribosomal structure, and biogenesis; K: Transcription; L: Replication, recombination, and repair; M: Cell wall/membrane/envelope biogenesis; N: Cell motility; O: Posttranslational modification, protein turnover, chaperones; P: Inorganic ion transport and metabolism; Q: Secondary metabolites biosynthesis, transport, and catabolism; R: General function prediction only; S: Function unknown; T: Signal transduction mechanisms; U: Intracellular trafficking, secretion, and vesicular transport; V: Defense mechanisms; W: Extracellular structures; X: Mobilome: prophages and transposons; Z: Cytoskeleton.

FIGURE S2 | Verification by PCR amplification of four Kangiella species, including genes involved in sulfite reduction (A,D) corresponding to Kkor_0726 to Kkor_0729 in the K. koreensis DSM 16069, genes involved in phenylalanine biosynthesis (B,E) corresponding to Kkor_1584 and Kkor_1585 in K. koreensis DSM 16069, genes involved in arginine biosynthesis (C,F, shown in green color) corresponding to Kkor_0543 to Kkor_0548 in K. koreensis DSM 16069, and genes of cytochrome c oxidase subunits $(\mathbf{C}, \mathbf{G}$, shown in red color) corresponding to Kkor_0534 to Kkor_0542 in K. koreensis DSM 16069. Ka, K. aquimarina DSM 16071; Kk, K. koreensis DSM 16069; Kg, K. geojedonensis KCTC 23420; Kp,

K. profundi FT102. The numbers in (A-C) represent genes listed in the following:

\section{REFERENCES}

Ahn, J., Park, J.-W., Mcconnell, J. A., Ahn, Y.-B., and Häggblom, M. M. (2011). Kangiella spongicola sp. nov., a halophilic marine bacterium isolated from the sponge Chondrilla nucula. Int. J. Syst. Evol. Microbiol. 61, 961-964. doi: 10.1099/ ijs.0.021733-0

Azam, F., and Malfatti, F. (2007). Microbial structuring of marine ecosystems. Nat. Rev. Microbiol. 5, 782-791. doi: 10.1038/nrmicro1747

Aziz, R. K., Bartels, D., Best, A. A., Dejongh, M., Disz, T., Edwards, R. A., et al. (2008). The RAST Server: rapid annotations using subsystems technology. BMC Genomics 9:75. doi: 10.1186/1471-2164-9-75

Cao, Y., Chastain, R. A., Eloe, E. A., Nogi, Y., Kato, C., and Bartlett, D. H. (2014). Novel psychropiezophilic Oceanospirillales species Profundimonas piezophila gen. nov., sp. nov., isolated from the deep-sea environment of the Puerto Rico trench. Appl. Environ. Microbiol. 80, 54-60. doi: 10.1128/AEM.02288-13

Chen, M., Xiao, X., Wang, P., Zeng, X., and Wang, F. (2005). Arthrobacter ardleyensis sp. nov., isolated from Antarctic lake sediment and deepsea sediment. Arch. Microbiol. 183, 301-305. doi: 10.1007/s00203-0050772-y

Choe, H., Kim, S., Oh, J., Nasir, A., Kim, B. K., and Kim, K. M. (2015). Complete genome of Kangiella geojedonensis KCTC 23420(T), putative evidence for recent genome reduction in marine environments. Mar. Genomics 24(Pt 3), 215-217. doi: 10.1016/j.margen.2015.05.015
1: cysJ, FAD-binding domain protein; 2: sulfite reductase subunit beta; 3 : $\mathrm{cysH}$, phosphoadenosine phosphosulfate reductase; 4: cysG, uroporphyrin-III C-methyltransferase; 5: prephenate dehydratase; 6:

phospho-2-dehydro-3-deoxyheptonate aldolase; 7: cytochrome-c oxidase, cbb3-type subunit I; 8: cytochrome-c oxidase, cbb3-type subunit II; 9: CcoQ/FixQ family Cbb3-type cytochrome c oxidase assembly chaperone; 10: cytochrome-c oxidase, cbb3-type subunit III; 11: cytochrome c oxidase accessory protein CcoG; 12: nitrogen fixation protein FixH; 13: cadmium-translocating P-type ATPase; 14: cbb3-type cytochrome oxidase assembly protein CcoS; 15: sulfite exporter TauE/SafE family protein; 16: $\mathrm{N}$-acetylornithine carbamoyltransferase; 17: argininosuccinate synthase; 18: acetylornithine deacetylase; 19: acetylglutamate kinase; 20: N-acetyl-gamma-glutamyl-phosphate reductase; 21:

argininosuccinate lyase; 22: oxygen-independent coproporphyrinogen III.

FIGURE S3 | Peptidase gene clusters (S8 FML) in the five Kangiella species. Arrows shown in black represent non-peptidase conserved genes. Arrows in the other colors represent the genes of different serine proteases belonging to the S8 family in the five Kangiella strains, including KangOF190 (orange), KangOF9 (green), KangOF8 (pink), and KangOF2260 (blue). Highlighted regions within the arrow (in red color) show the PKD domain.

TABLE S1 | Number of genes belonging to each cluster of orthologous genes (COG) category in all 23 strains used in the present study.

TABLE S2 | Orthologous families of the five Kangiella strains.

TABLE S3 | Orthologous families having paralogs in the core genome of the five Kangiella strains.

TABLE S4 | Cluster of orthologous genes (COG) distribution of the Kangiella pangenome.

TABLE S5 | Genomic features of the 23 Oceanospirillales strains used herein.

TABLE S6 | Orthologous families of the 23 Oceanospirillales strains.

TABLE S7 | Kangiella-specific missing GOs.

TABLE S8 | Number of SignalP-fused proteases identified in all 23 genomes.

MATERIAL S1 | ".ab1" files and PCR primers for genomic gap filling.

MATERIAL S2 | Raw gel electrophoresis maps and PCR primers corresponding to Supplementary Figure S3.

MATERIAL S3 | Peptidase genes predicted with MEROPS batch BLAST and SignalP prediction of 23 genomes corresponding to Supplementary Table $\mathbf{S 8}$

Choi, Y. U., Kwon, Y. K., Ye, B. R., Hyun, J. H., Heo, S. J., Affan, A., et al. (2012). Draft genome sequence of Marinobacterium stanieri S30, a strain isolated from a coastal lagoon in Chuuk state in Micronesia. J. Bacteriol. 194:1260. doi: 10. 1128/JB.06703-11

Darling, A. C. E., Mau, B., Blattner, F. R., and Perna, N. T. (2004). Mauve: multiple alignment of conserved genomic sequence with rearrangements. Genome Res. 14, 1394-1403. doi: 10.1101/gr.2289704

Delcher, A. L., Harmon, D., Kasif, S., White, O., and Salzberg, S. L. (1999). Improved microbial gene identification with GLIMMER. Nucleic Acids Res. 27, 4636-4641. doi: 10.1093/nar/27.23.4636

DeLong, E. F., and Karl, D. M. (2005). Genomic perspectives in microbial oceanography. Nature 437, 336-342. doi: 10.1038/nature04157

Gao, F., and Zhang, C. T. (2008). Ori-Finder: a web-based system for finding oriCs in unannotated bacterial genomes. BMC Bioinformatics 9:79. doi: 10.1186/14712105-9-79

Han, C., Sikorski, J., Lapidus, A., Nolan, M., Glavina Del Rio, T., Tice, H., et al. (2009). Complete genome sequence of Kangiella koreensis type strain (SW-125(T)). Stand. Genomic Sci. 1, 226-233. doi: 10.4056/sigs. 36635

Jean, W. D., Huang, S.-P., Chen, J.-S., and Shieh, W. Y. (2012). Kangiella taiwanensis sp. nov. and Kangiella marina sp. nov., marine bacteria isolated from shallow coastal water. Int. J. Syst. Evol. Microbiol. 62, 2229-2234. doi: 10.1099/ijs.0.037010-0 
Jisha, V. N., Smitha, R. B., Pradeep, S., Sreedevi, S., Unni, K. N., Sajith, S., et al. (2013). Versatility of microbial proteases. Adv. Enzyme Res. 1, 39-51. doi: 10.4236/aer.2013.13005

Jung, G., Ueno, H., and Hayashi, R. (1998). Proton-relay system of carboxypeptidase $\mathrm{Y}$ as a sole catalytic site: studies on mutagenic replacement of his 397. J. Biochem. 124, 446-450. doi: 10.1093/oxfordjournals.jbchem.a022133

Kim, J.-H., Ward, A. C., and Kim, W. (2015). Kangiella chungangensis sp. nov. isolated from a marine sand. Antonie Van Leeuwenhoek 107, 1291-1298. doi: 10.1007/s10482-015-0423-5

Lagesen, K., Hallin, P., Rodland, E. A., Staerfeldt, H. H., Rognes, T., and Ussery, D. W. (2007). RNAmmer: consistent and rapid annotation of ribosomal RNA genes. Nucleic Acids Res. 35, 3100-3108. doi: 10.1093/nar/ gkm160

Lee, S. Y., Park, S., Oh, T. K., and Yoon, J. H. (2013). Kangiella sediminilitoris sp. nov., isolated from a tidal flat sediment. Int. J. Syst. Evol. Microbiol. 63, 1001-1006. doi: 10.1099/ijs.0.040691-0

Lerat, E., Daubin, V., Ochman, H., and Moran, N. A. (2005). Evolutionary origins of genomic repertoires in bacteria. PLoS Biol. 3:e130. doi: 10.1371/journal.pbio. 0030130

Li, L., Stoeckert, C. J. Jr., and Roos, D. S. (2003). OrthoMCL: identification of ortholog groups for eukaryotic genomes. Genome Res. 13, 2178-2189. doi: $10.1101 /$ gr.1224503

Lowe, T. M., and Eddy, S. R. (1997). tRNAscan-SE: a program for improved detection of transfer RNA genes in genomic sequence. Nucleic Acids Res. 25, 955-964. doi: 10.1093/nar/25.5.0955

Luo, H., Friedman, R., Tang, J., and Hughes, A. L. (2011). Genome reduction by deletion of paralogs in the marine cyanobacterium Prochlorococcus. Mol. Biol. Evol. 28, 2751-2760. doi: 10.1093/molbev/msr081

Petersen, T. N., Brunak, S., Von Heijne, G., and Nielsen, H. (2011). SignalP 4.0: discriminating signal peptides from transmembrane regions. Nat. Methods 8 , 785-786. doi: 10.1038/nmeth.1701

Rawlings, N. D., Barrett, A. J., and Finn, R. (2016). Twenty years of the MEROPS database of proteolytic enzymes, their substrates and inhibitors. Nucleic Acids Res. 44, D343-D350. doi: 10.1093/nar/gkv1118

Rawlings, N. D., Polgar, L., and Barrett, A. J. (1991). A new family of serinetype peptidases related to prolyl oligopeptidase. Biochem. J. 279(Pt 3), 907-908. doi: 10.1042/bj2790907

Romanenko, L. A., Tanaka, N., Frolova, G. M., and Mikhailov, V. V. (2010). Kangiella japonica sp. nov., isolated from a marine environment. Int. J. Syst. Evol. Microbiol. 60, 2583-2586. doi: 10.1099/ijs.0.017087-0

Tao, C., Li, H., Huang, W., Han, X., Wu, G., Su, X., et al. (2011). Mineralogical and geochemical features of sulfide chimneys from the $49^{\circ} 39^{\prime} \mathrm{E}$ hydrothermal field on the Southwest Indian Ridge and their geological inferences. Chin. Sci. Bull. 56, 2828-2838. doi: 10.1007/s11434-011-4619-4
Tao, C., Lin, J., Guo, S., Chen, Y. J., Wu, G., Han, X., et al. (2012). First active hydrothermal vents on an ultraslow-spreading center: Southwest Indian Ridge. Geology 40, 47-50. doi: 10.1130/G32389.1

Wang, G., Tang, M., Wu, H., Dai, S., Li, T., Chen, C., et al. (2015). Aliikangiella marina gen. nov., sp. nov., a marine bacterium from the culture broth of Picochlorum sp. 122, and proposal of Kangiellaceae fam. nov. in the order Oceanospirillales. Int. J. Syst. Evol. Microbiol. 65, 4488-4494. doi: 10.1099/ijsem. 0.000601

Widdel, F., Boetius, A., and Rabus, R. (2006). "Anaerobic biodegradation of hydrocarbons including methane," in The Prokaryotes: Ecophysiology and Biochemistry, Vol. 2, eds M. Dworkin, S. Falkow, E. Rosenberg, K.-H. Schleifer, and E. Stackebrandt (New York, NY: Springer), 1028-1049.

Xu, F. D., Li, X. G., Xiao, X., and Xu, J. (2015). Kangiella profundi sp nov., isolated from deep-sea sediment. Int. J. Syst. Evol. Microbiol. 65, 2315-2319. doi: 10.1099/ijs.0.000257

Xu, L., Wang, J., Lu, Y., and Xu, J. (2018). The growth, extracellular protease activity and the transcription of protease coding gene of four Kangiella strains affected by the major metal ions in the sea water. Microbiol. China 45, 1-13.

Yamagata, H., Masuzawa, T., Nagaoka, Y., Ohnishi, T., and Iwasaki, T. (1994). Cucumisin, a serine protease from melon fruits, shares structural homology with subtilisin and is generated from a large precursor. J. Biol. Chem. 269, 32725-32731.

Yoon, J. H., Kang, S. J., Lee, S. Y., Lee, J. S., and Oh, T. K. (2012). Kangiella geojedonensis sp. nov., isolated from seawater. Int. J. Syst. Evol. Microbiol. 62, 511-514. doi: 10.1099/ijs.0.029314-0

Yoon, J. H., Oh, T. K., and Park, Y. H. (2004). Kangiella koreensis gen. nov., sp. nov. and Kangiella aquimarina sp. nov., isolated from a tidal flat of the Yellow Sea in Korea. Int. J. Syst. Evol. Microbiol. 54, 1829-1835. doi: 10.1099/ijs.0. 63156-0

Zerbino, D. R., and Birney, E. (2008). Velvet: algorithms for de novo short read assembly using de Bruijn graphs. Genome Res. 18, 821-829. doi: 10.1101/gr. 074492.107

Conflict of Interest Statement: The authors declare that the research was conducted in the absence of any commercial or financial relationships that could be construed as a potential conflict of interest.

Copyright (c) 2018 Wang, Lu, Nawaz and Xu. This is an open-access article distributed under the terms of the Creative Commons Attribution License (CC BY). The use, distribution or reproduction in other forums is permitted, provided the original author(s) and the copyright owner are credited and that the original publication in this journal is cited, in accordance with accepted academic practice. No use, distribution or reproduction is permitted which does not comply with these terms. 